\title{
JEAN-ETIENNE BERTIN Sur les Q-anneaux de Matlis
}

Publications des séminaires de mathématiques et informatique de Rennes, 1976, fascicule 4

«Colloque d'algèbre commutative », , exp. no 10, p. 1-10

$<$ http://www.numdam.org/item?id=PSMIR_1976__4_A6_0>

(C) Département de mathématiques et informatique, université de Rennes, 1976, tous droits réservés.

L'accès aux archives de la série « Publications mathématiques et informatiques de Rennes » implique l'accord avec les conditions générales d'utilisation (http://www.numdam.org/conditions). Toute utilisation commerciale ou impression systématique est constitutive d'une infraction pénale. Toute copie ou impression de ce fichier doit contenir la présente mention de copyright.

\section{Numdam}

Article numérisé dans le cadre du programme Numérisation de documents anciens mathématiques http://www.numdam.org/ 
par

Jean-Etienne BERTIN

\section{GENERALTTES}

\section{Notations}

Dans tout ce qui suit, $R$ désigne un anneau commutatif unitaire intègre qui n'est pas un corps, $Q$ son corps des fractions, et $K$ le $R$-module $Q / R$. On note $H L^{\prime}$ anneau $\operatorname{Hom}_{R}(K, K)$. Si $M$ est un R-module sans torsion, on note Q rg(M) Ia dimension sur $Q$ de $I$ 'espace vectoriel $M a_{R} Q$.

\section{Introduction}

Le point de dêpart de la théorie des Q-anneaux se situe dans l'étude de la cohomologie singulière des groupes abéliens, pour laquelle Kan et Whitehead [3] ont montré qu'il n'existe pas de groupe abêlien.M tel que $\operatorname{Hom}_{Z}(M, Z)=0$ et $\operatorname{Ext}_{Z}^{1}(M, Z)=Q$. Plus généralement, existe-t-il un R-module $M$ tel que $\operatorname{Hom}_{R}(M, R)=0$, et que $\operatorname{Ext}_{R}^{1}(M, R)$ soit $R$-isomorphe à un Q-espace vectoriel non nul de dimension finie ? On montre aisément qu'il en est

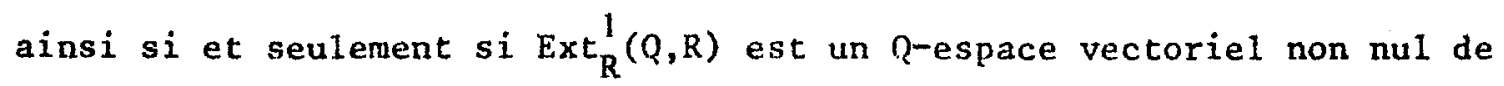
dimension finie.

Définition. 1: On dit que $R$ est un Q-anneau si $\operatorname{Ext}_{R}^{1}(Q, R)$ est un espace vectoriel de dimension finie sur $Q$. Plus précisément, étant donné un entier $n$ positif ou nul, on dit que $R$ est un $Q^{n}$-anneau si $\operatorname{Ext}_{R}^{1}(Q, R) \simeq Q^{n}$.

Proposition 1: Etant donné $n \in \mathbb{N}$, les conditions suivantes sont équivalentes :

(i) $R$ est un $Q^{n}$-anneau.

(ii) $Q-r g(H)=n+1$.

(iii) $H / R \simeq Q^{n}$. 
Cela rósulte de la suite exacte suivante ([5], th. 10): $\mathrm{O} \longrightarrow \mathrm{R} \longrightarrow \mathrm{H} \longrightarrow \operatorname{Ext}_{\mathrm{R}}^{\prime}(\mathrm{Q}, \mathrm{R}) \longrightarrow \mathrm{O}$.

Remarque 1: Munissons $\mathrm{R}$ de la topologie linéaire pour laquelle les idéaux non nuls de $R$ forment un système fondamental de voisinages de 0 . Alors $H$ s'identifie au complété de $R$ pour cette topologie. Lorsque $R$ est un anneau de valuation, la topologie dêcrite ci-dessus et la topologie définie par la valuation coincident, de sorte que tout anneau de valuation complet est un $Q^{\circ}$-anneau (pour plus de détails, voir [4])

Exemple 1 : Soit p un nombre premier. Nagata ( $[8]$, E 3.3) a donnê un exemple d'anneau de valuation discrète $R$ ayant les propriétés suivantes :

1) Le corps des fractions $Q$ de $R$ est de caractéristique $p$.

2) Si L désigne le corps des fractions du complêté $H$ de $R$, on a $[L: O]=\mathrm{P}$, et $L$ est une extension radicielle de $Q$.

Alors $R$ est un $Q^{P^{-1}}$-anneau. Pour tout entier positif $s$, cet exeraple peut être modifié de façon a fournir un $0^{\mathrm{p}^{\mathrm{s}}-1}$-anneau de valuation discrète [2].

Remarque 2 : Jensen [2] a annoncé le résultat suivant : $I$ 'ensemble des cardinaux de la forme $\left[\operatorname{Ext}_{R}^{l}(\Omega, R)\right.$ : 0$]$, où $R$ est un anneau intègre noethérien de dimension 1 analytiquement non-ramifié en un idéal maximal au moins, et $\mathbb{Q}$ son corps des fractions se compose de tous les cardinaux infinis, de 0 et des nombres de 1 a forme $\mathrm{p}^{s^{-1}}$, où $p$ est un nombre premier, et $s$ un entier positif. Cet ensemble reste inchange si on impose à $R$ d'être un anneau de valuation discrète.

\section{SOUS-MODULES h-DIVISIBLES DE-K ET IDEAUX PREMXERS DE H.}

Dans ce numēro, $R$ dësigne un $Q^{n}$-anneau, pour $n \in \mathbb{N}$. Les résultats de ce numëro ont étẻ démontrés par Matlis dans le cas où $n=1[6]$. 
Rappelons que $H$ est un anneau commutatif ([5], th. 10), et notons Ass (H) L'ensemble des jdêaux premiers associés à $H$ (c'est-à-dire de la forme $(0: f)$ pour un élëment $E$ de $\operatorname{l}$ convenable).

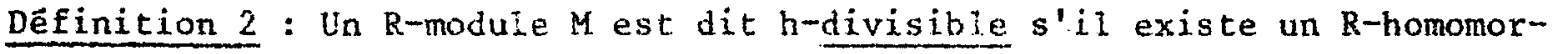
phisme surjectif $N \rightarrow M$, oii $N$ est un n-module.

Proposition 2 : Soit $\mathrm{E}$ un êlément de H. Les conditions suivantes sont équivalentes :

(i) f est diviseur de zéro dans H.

(ii) $\mathrm{f}(\mathrm{K}) \neq \mathrm{K}$.

(iii) $\operatorname{Hom}_{R}(Q, \operatorname{Ker}(f)) \neq 0$.

En particulier, $H$ est intègre si et seulement si $O$ et $K$ sont les seuls sous-modules h-divisibles de $K$.

Theorème 1 : Soit $\mathrm{P}$ un idêal premier faiblement associê à H (c'est-à-dire tel qu'il existe $f \in A$ pour lequel $P$ soit un idëal premier minimal parmi ceux qui contiennent $(O: F))$. Alors $P \in$ Ass $(H)$, et $i l$ existe un sous-module h-divisible $D$ de $K$ tel que $P=\operatorname{Hom}_{R}(K, D)$.

Corollaire 1 : Supposons H rẻduit; alors Ass(H) a au plus $n+1$ élément, et tout êlëment de Ass(H) est un idêal premier minimal de $H$.

Corollaire 2 : Supposons H non rêduit; alors Ass(H) a au plus $\mathrm{n}^{2}$ élêments.

Proposition 3: Tout idéal J de $\mathrm{H}$ tel que $\mathrm{Q}-\mathrm{rg}(\mathrm{J}) \leq \mathrm{n}$ est inclus dans un êlément de Ass(H). A fortiori tout idéal $J$ de $H$ tel que $J \quad R=0$ est inclus dans un êlêment de Ass(H).

Proposition 4 : Soit L I'anneau total des fractions de $H ;$ alors $L \cong H{ }_{R} Q$. 


\section{UN CRITERE POUR QUE H SOIT UN ANNEAU RFDUTT}

Notons $r$ la clôture intégrale de l'anneau intègre $R$. Supposons d'abord ue $R$ soit un annean local noethërien intègre de dimension 1 d'idéal maximal m ; alors $H$ n'est autre que le complété de $R$ pour la topologie m-adique, et Krul1 a montré que $\mathrm{H}$ est rêduit si et seulement si $\Gamma$ est un R-module de type fini (ce qui a lieu si et seulement si $\operatorname{Hom}_{R}(O, \Gamma / R)=0$ ).

Dans le cas d'un anneau intègre quelconque $R$, Matlis a montré que si $\operatorname{Hom}_{R}(Q, \Gamma / R)=0$, alors $H$ est reduit $([4], 8.10)$. Dans le cas d'un $Q-a n n e a 1$, on a la réciproque :

Théorème 2 : Soient $R$ un Q-anneau, et $\Gamma$ sa clôture intégrale. Alors $H$ est réduit si et seulement si. $\operatorname{Hom}_{R}(Q, \Gamma / R)=0$.

Supposons donc $H$ réduit, et soit $P$ Ess(H). Alors $P=\operatorname{Hom}_{R}(K, D)$, où $D$ est un sous-module $h$-divisible de $K$. Soient $A$ le sous-R-module de $Q$ tel que $R \subset A \subset Q$, et $A / R=D$; alors $A$ est un sous-anneau de $Q([6], 1.8)$. II existe donc un anneau de valuation $V_{P}$ de $Q$ tel que $A \subset V_{P}$. Posons $P^{\prime}=\operatorname{Hom}_{R}\left(R, v_{P} / R\right)$. Alors $P \subset P^{\prime}$ et $P^{\prime} \cap R=0$. II résulte alors de la proposition 3 et du corollaire 1 au théorème 1 que $P=p^{\prime}$. On a donc : $\operatorname{Hom}_{R}(K, r / R) \subset \operatorname{Hom}_{R}\left(K, \underset{P \in A s s(H)}{ } V_{P} / R\right)=\bigcap_{P \in A s s(H)} P=0$ puisque $H$ est réduit ([1], chap. IV, 1 , exercice 17). Puisque $\Gamma / R$ est un $R$-module de torsion, on a donc $\operatorname{Hom}_{R}(Q, \Gamma / R)=0$.

\section{MODULES ETRANGERS}

Dans ce numéro, A désigne un anneau unitaire, non nécessairement commutatif.

Définition 3 : Soient $A$ un anneau, $M$ un A-module, et $M_{1}, \ldots, M_{n}$ une suite de sous-A-modules de $M$. On dit que $M_{1}, \ldots, M_{n}$ est une suite de sous-A-modules 
étrangers de $M$ si pour $i$ de 2 ì $n$, on a

$$
M_{i}+\prod_{1 \leq j<i} M_{j}=M .
$$

Lemme : Soient A un anneau, $M$ un $A$-nodule, $M_{1}, \ldots M_{n}$ une suite de sous-Amodules étrangers de $M$. Alots:

(i) On a une suite exacte :

$$
0 \longrightarrow \bigcap_{i=1}^{n} M_{i} \longrightarrow M-\underline{M} \rightarrow \sum_{i=1}^{n}\left(M / M_{i}\right) \longrightarrow \cdots
$$

où $p$ est la somme des projections canoniques $M \rightarrow M / M_{i}$.

(ii) On a une suite exacte:

$0 \longrightarrow \bigcap_{i=1}^{n} M_{i} \stackrel{\delta}{\rightarrow} \bigoplus_{i=1}^{n} M_{i} \stackrel{a}{\longrightarrow} M^{n-1} \longrightarrow 0$

où $\delta$ est 1 'homomorphisme diagonal, et où $a\left(x_{1}, \ldots, x_{n}\right)=\left(x_{1}-x_{2}, \ldots, x_{1}-x_{n}\right)$ pour tout $\left(x_{1}, \ldots, x_{n}\right) \in \bigoplus_{i=1}^{n} M_{i}$.

Exemple 2 : Soit $V_{1}, \ldots, v_{n}$ une suite d'anneaux de valuation deux à deux indépendants d'un corps $R$, et posons $R=\bigcap_{i=1}^{n} v_{i}$. Alors $v_{1}, \ldots, v_{n}$ est une suite de sous-R-modules ëtrangers de $Q$.

\section{ANNEAUX SPECIAUX}

Les résultats de ce numêro et des deux suivants relatifs aux $Q^{1}$-anneaux ont étê démontrês par Mat $I$ is $[6]$.

Définition 4 : Soit $R$ un $Q$-anneau [resp. un $Q^{n}$-anneau]. On dit que $R$ est un $Q_{\mathrm{T}}$-anneau [resp. $Q_{I^{n}}^{n}$ anneau] si H est intègre, un $Q_{R^{-a n n e a u}}$ [resp. $Q_{R}^{\mathfrak{n}}-$ anneau] si $\mathrm{H}$ est réduit sans être intègre, et un $Q_{N}$-anneau $\left[\right.$ resp. $Q_{N}^{n}$-anneau] si $H \cdot n^{\prime}$ est pas réduit.

Dëfinition 5 : Un anneau intègre R est dit special s'il existe des sousanneaux $A_{1}, \ldots, A_{p}$ de $Q$ vérifiant les conditions suivantes : 
1) On a $R \varsubsetneqq A_{i} \subsetneq Q$ pour ide 1 a p, et $R=\bigcap_{i=1}^{p} A_{i}$.

2) $A_{1}, \ldots, A_{p}$ est une suite de sous-R-modules étrangers de $Q$.

3) Pour tout $i$ de 1 à $p, A_{i}$ est un $Q_{T}$-anneau.

Proposition 5 : Un annear $R$ est un $Q_{R}$-anneau si et seulement $s^{\prime} i l$ existe un anneau spécial $S$ tel. que $R \subset S \subset Q$ et gu'il existe $r \in R-\{0\}$ tel que $r(S / R)=0$. En particulier, tout anneau spécial est un $Q_{R}$-anneau.

Détinition 6: On dit que $\mathrm{R}$ est h-local s'il vérifie les conditions suivantes:

(i) Tout idéal premier non nuI de $R$ est inclus dans un seul idéal maximal de $R$.

(ii) Tout êlément non nu1 de $R$ n'appartient qu'à un nombre fini d'ideaux maximaux de $R$.

Par exemple, tout anneau intègre nothérien de dimension 1 est h-local.

Theorème 3 : Soit $R$ un $Q_{R}^{n}$-anneau, oì $n$ est un entier positif. Soient $\mathrm{P}_{1}, \ldots, \mathrm{P}_{\mathrm{p}}$ les idéaux premiers associés $\mathrm{a} \mathrm{H}$. Pour $\mathrm{i}$ de $1 \mathrm{a} \mathrm{p}$, notons $\mathrm{A}_{i}$ le sous-R-module de $Q$ tel que $R \subset A_{i}$, que $A_{i} / R$ soit un sous-R-module $h$-divisible de $K$ et que $P_{i}=$ Fom $_{R}\left(K, A_{i} / R\right)$. Posons $S=\bigcap_{i=1}^{p} A_{i}$; alors :

(i) Pour $i$ de 1 à $p, A_{i}$ est un $Q_{I}{ }{ }$-anneau, l'entier $d_{i}$ vérifiant $0-d_{i}-n+1-p,-t \sum_{n=1}^{p} d_{i}=n+1-p$.

(ii) Pour $i$ de 1 à $p$, on a un isomorphisme $d$ 'anneaux de $H / P_{i}$ sur $H_{0 m} A_{i}$ $\left(Q / A_{i}, Q / A_{i}\right)$.

(iii) $S$ est un $Q^{n}$-anneau spécial, entier sur $R$, et il existe $r \in R-\{0\}$ tel que $r(S / R)=0$.

(iv) La clôture intégrale de $\mathrm{R}$ est l'intersection des clôtures intégrales des $A_{i}$, pour $i$ de 1 à $p$.

(v) Pour $i$ de 1 a $p$, notons $L_{i}$ le corps des fractions de $H / P_{i}$, et soit $L$ I'anneau total des fractions de $H$. Aiors $L=\underset{i=1}{p} I_{i}$. 
(vi) Soit $M$ un idêal maximal de $n$; alors $s$ a au plus $p$ idéaux maximaux au-dessus de $M$, et $S_{M}$ est un anneau h-local ayant au plus $p$ idêaux maximaux.

(vii) $R$ est spécial si et seulement si $S$ a un unique idéal maximal au-dessus de chacun des idéaux maximaux de $R$, auquel cas $R=S$.

(viii) Si $R$ est local, alors s est h-local et possìde exactement $p$ idéaux maximaux $N_{1}, \ldots, N_{p}$ qu'on peut numeroter de sorte qu'on ait $A_{i}=S_{\mathbb{N}_{i}}$ pour $i$ de 1 ì $p$.

Corollaire : Un anneau local intègre unibranche ne peut être un $Q_{R}$-anneau.

Proposition 6 : Un anneau intègre $\mathrm{R}$ est h-local et spécial si et seulement si. c'est un $Q^{\mathrm{n}}$-anneau non local (pour $\mathrm{n}$ entier positif convenable) et si, pour tout idéal maxinal $M$ de $R, R_{M}$ est un $Q_{T}$-anneau. Si tel est le cas, $R$ a au plus $n+1$ idéaux maximaux.

6. EXTENSIONS DE $Q_{I}$-anneaux.

Proposition 7 : Soit $R$ un $Q_{T}^{n}$-anneau, pour $n \in \mathbb{N}$. Notons $L$ le corps des fractions de H. Les conditions suivantes sont équivalentes :

(i) H est entier sur R.

(ii) $L$ est une extension radicielle de $Q$.

Lorsque ces conditions sont vêrifiées, soit $x \in H-R$ tel que $Q(x)=L$. Nlors $R[x]$ est un $L_{N}^{n}$-anneau.

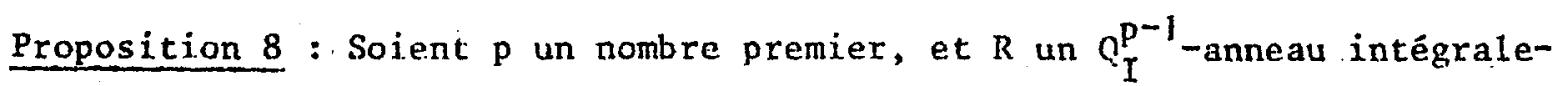
ment clos; notons $\Lambda$ la fermeture intégrale de $R$ dans le corps des fractions $L$ de $H$. Si $L$ est une extension séparable de $Q$, alors $\Lambda$ est un $L_{R}^{p^{-1}}$-anneau spécial. 


\section{CAS OU R EST NOETHERIEN DE DT'ENSTON 1}

Soit $\mathrm{R}$ un Q-anneau noethérien de dimension 1 ; d'après Jensen [2], $R$ est un anneau local. Si de plus, $R$ est un $Q_{I}^{n}$-anneau pour $n \in \mathbb{N}$, d'après Matlis $([7], 7.1)$, la clôture intégraie $Y$ de $R$ est un $Q_{I}^{n}$-anneau de valuation discrète et un R-module de type fini. Si de plus, $R$ est un $Q_{I}^{n}$-anneau pour n entier positif, d'après Jensen [2], le corps résiduel de $R$ n'est pas parfait.

Proposition 9.: Un Q-anneau $R$ noethérien de dimension 1 ne peut être un $Q_{R}-$ anneau. Autrement dit, $K$ est analytiquement non ramifié si et seulement s'il est analytiquement irréductible.

Thëorème 4 : Soient $\mathrm{p}$ un nombre premier, et $\mathrm{R}$ un $\mathrm{Q}_{\mathrm{I}}^{\mathrm{p}-1}$-anneau noethérien de dimension 1. Alors $Q$ est de caracteristique $p$, et le corps des fractions $L$ de $H$ est une extension radicielle de $Q$.

\section{EXEMPLES}

Exemple 3 : Soient $p$ un nombre premier, et $R$ le $Q_{I}^{p-1}$-anneau de l'exemple 1 . Soit $x \in H \sim R$; alors $Q(x)$ est égal au corps des fractions $L$ de $H$, et d'après Ia proposition $7, R[x]$ est un $L_{N}^{p-1}$-anneau noethërien de dimension 1 .

Exemple 4 (Jensen) : Soient $p$ un nombre premier, et $Q_{p}$ le corps des fractions de $I$ 'anneau $\hat{\mathbf{z}}_{\mathrm{p}}$ des entiers p-adiques. Alors le complété $\hat{\bar{Q}}_{p}$ de la clôture a1gébrique $\bar{Q}_{p}$ de $Q_{p}$ pour la valuation v prolongeant à $\bar{Q}_{p}$ la valuation $p$-adique est un corps $Q-i$ somorphe à c. Donc I'anneau de valuation $v_{p}$ de $\hat{Q}_{p}$ pour $v$ est un anneau de valuation complet de hauteur 1 de $c$ (et donc un $C_{I}^{o}$-anneau) tel que $v_{p} \cap Q=x_{p}$

Exemple 5 : Soient $p_{1}, \ldots, p_{d}$ des nombres premiers deux à deux distincts, où d 2. Avec les notations de 1. exemple $4, v_{p_{1}} \cap \ldots \cap v_{p_{d}}$ est un $a_{R}^{d-1}$ - anneau spécial. 
Exemple 6 : Soient $p_{1}, \ldots, p_{d}$ des nombres premiers deux à deux distincts, on $d \geq 2$. Avec ies notations de l'exemple $4, v_{p_{1}} \cap \ldots \cap v_{p_{d}} \cap \mathbb{R}$ est un $R_{R}^{2 d-1}$ anneau spécial.

Exemple 7 (Jensen) : Soient $p$ un nombre premier, et $V_{p} 1$ 'anneau de l'exemple 4. Posons $R=V_{p} \cap \mathbb{R} ;$ alors $R$ est un $\mathbb{R}_{I}^{l}$-anneau pour leque $1 H=V_{p}$, et $1 a$ fermeture intégrale $A$ de $R$ dans $\mathbb{C}$ est un $\mathbb{C}_{R}^{b}$-anneau spécial. 


\section{BIBL IOGRAPHTE}

[1] BOURBAKI N. Algèbre commutative. Hermann, Paris.

[2] JENSEN C.U. On $\operatorname{Ext}_{R}(A, R)$ for torsion-free A, Bull. Amer. Math. Soc. 78 (1972) 831-834.

[3] KAN D.M. - WUITEHEAD G.W. On the realizability of singular cohomology groups, Proc. Amer. Math. Soc. 12 (1961) 24-25.

[4] Matlis E. Cotorsion modules Mern. Amer. Math. Soc. $n^{\circ} 49$ (1964).

[5] MATLIS E. Torsion-free modules the University of Chicago Press (1972).

[6] MATLIS E. The theory of Q-rings Troms. Amer. Math. Soc. 187 (1974) 147-181.

[7] MATLIS E. 1-dimensional Cohen-Macaulay rings Lect. notes in Math. $n^{\circ} 327$ Springer Verlag (1973).

[8] NAGATA M. Local rings. Interscience Tracts in Pure an App1. Math. $n^{\circ} 13$ Interscience New-York (1962). 